Check for updates

Cite this: Mater. Chem. Front., 2021, 5, 8151

Received 10th June 2021

Accepted 4th October 2021

DOI: 10.1039/d1qm00855b

rsc.li/frontiers-materials

\title{
Amphiphilic-like carbon dots as antitumoral drug vehicles and phototherapeutical agents $\dagger$
}

\author{
Mariano Ortega-Muñoz, ${ }^{a}$ Paula Vargas-Navarro, ${ }^{a}$ Simona Plesselova, ${ }^{b}$ \\ M. Dolores Giron-Gonzalez, (D) ${ }^{b}$ Guillermo R. Iglesias, (D) ${ }^{c}$ Rafael Salto-Gonzalez, \\ Fernando Hernandez-Mateo, (D) a Angel V. Delgado, (D) ${ }^{c}$ F. Javier Lopez-Jaramillo (D) *a \\ and Francisco Santoyo-Gonzalez (D) *a
}

\begin{abstract}
Water-insoluble carbon dots are recognized as promising materials, although their applications in nanomedicine are rarely explored, despite their lipophilic character and foreseen compatibility with biological membranes. In this article, we exploit the anhydride functionalization of carbon dots obtained by thermolysis of citric acid to synthesize amphiphilic-like carbon dots (LCDs) by reaction with alkyl amines. A differential feature of this approach is that the hydrophobicity of LCDs is a balance between the ionization of the carboxylic groups resulting from the reaction and the hydrophobicity from the grafted amines. The alkyl chains allow LCDs to entrap hydrophobic molecules and the ionization of the carboxylic groups increases the hydrosolubility, permitting the transfer between organic and aqueous phases. The biomedical interest of these features is illustrated by analyzing the application of LCDs as carriers of the drug campothecin and their evaluation on a battery of cancer cell lines, as well as the transformation of LCDs into a phototherapeutic agent by the formation of a complex with IR780 dye. Results demonstrate that LCDs behave as nanocarriers in a manner that resembles other supramolecular hosts with two differential features: (i) the length of the alkyl chains determines the size of the hosted guest, and (ii) the hydrosolubility of the complex can be modulated by $\mathrm{pH}$.
\end{abstract}

\section{Introduction}

Carbon dots (CDs) are nanosized carbon particles that, since their discovery in 2004, have attracted the attention of researchers from different fields for their potential applications due to their high photostability, tunable excitation and emission, low toxicity and high biocompatibility. ${ }^{1-4}$ The synthesis of CDs is well established. The preparation has been approached by bottom-up and top-down strategies using different sources of carbons, including biomass. ${ }^{5}$ CDs have been functionalized ${ }^{6}$ and doped with heteroatoms ${ }^{7}$ and metal ions to tune their properties and widen their applications. ${ }^{8}$ However, most CDs are hydrophilic and incompatible with organic solvents, limiting their application in organic-based electronic and energy storage devices or in synthesis and catalysis in organic solvents.

\footnotetext{
${ }^{a}$ Department of Organic Chemistry, Biotechnology Institute, Faculty of Sciences, University of Granada, Campus Fuentenueva s/n, 18071-Granada, Spain. E-mail: fjljara@ugr.es, fsantoyo@ugr.es

${ }^{b}$ Department of Biochemistry and Molecular Biology II, Faculty of Pharmacy, University of Granada, Campus Cartuja s/n, 18071-Granada, Spain c Department of Applied Physics, Faculty of Sciences, University of Granada, Campus Fuentenueva s/n, 18071-Granada, Spain

$\dagger$ Electronic supplementary information (ESI) available. See DOI: 10.1039/d1qm00855b
}

Reports on CDs that are soluble in non-aqueous solutions, termed hydrophobic CDs (hCDs), are still scarce. In general, the synthesis involves either the use of a water-insoluble binary mixture of a carbon source and a capping agent ${ }^{9-14}$ or the specific post-synthesis functionalization of hydrophilic CDs, ${ }^{15,16}$ although polysaccharides ${ }^{17}$ and microwave assisted synthesis ${ }^{18}$ have also been reported. hCDs bearing an aliphatic chain are an emerging subgroup that has focused the interest of researchers. Among the alkyl chains, 1-hexadecylamine is frequently used either as both carbon source and alkyl chain ${ }^{12}$ or in combination with citric acid (CA) ${ }^{19,20}$ or ascorbic acid ${ }^{21}$ as source of carbon.

Although the applications of hCDs are rarely detailed, they are recognized as promising materials and explored as sensors, ${ }^{22}$ fluorescent inks, ${ }^{23}$ nanoadditive for lubricants, ${ }^{24}$ or in lightemitting diodes. ${ }^{20}$ The compatibility of hCDs with biological membranes facilitates their traversing. ${ }^{14,25,26}$ This feature is attractive for biomedical applications, in particular in cancer, where drug resistance and low specificity urge the development of new drugs whose fate may be compromised by solubility, stability and clearance rate, as illustrated by the paradigmatic case of campothecin. ${ }^{27}$ Additionally, the side effects of anticancer agents are a challenge that demands new therapeutic approaches, among them the use of nanoparticles for photothermal therapy. ${ }^{28}$ In this context, heptamethyne dyes such as 
IR780 are interesting molecules not only for bioimaging and theranostics but as photothermal agents that yield a temperature increase and production of ROS upon illumination with near-infrared (NIR) light. ${ }^{29}$ However, poor solubility in biological fluids, toxicity and fast clearance are major limitations ${ }^{29}$ that have been partially overcome by encapsulating IR780 in diverse materials $^{30-35}$ or the production of CDs from IR780 and PEG by a solvothermal method. ${ }^{36}$

In this context, we describe in this work the thermolysis of CA in DMSO to obtain CDs functionalized with acid anhydride groups which, upon reaction with alkyl amines, are transformed into CDs (LCDs) presenting features that resemble those of amphibious ${ }^{17}$ and amphiplic ${ }^{15,24,26}$ CDs. LCDs bear both hydrophobic alkyl chains and carboxylic groups, the former making them suitable for hosting hydrophobic guests and the latter allowing the modulation of their hydrosolubility. As proof of concept of the biomedical applications of LCDs as carriers, the highly insoluble drug camptothecin and the photothermal and photodynamic therapeutic agent IR780 are examined as guests.

\section{Results and discussion}

\section{Design of the synthesis of LCDs}

hCDs are promising nanoparticles that are synthesized by the use of water-insoluble binary mixtures of a carbon source and a capping agent ${ }^{9-14}$ or by specific post-synthesis functionalization of hydrophilic CDs. ${ }^{15,17}$ We envisioned a two-step synthesis consisting of a first step of thermolysis of CA, to yield CDs functionalized with acid anhydride groups (AA-CDs), ${ }^{37}$ and a second step of reaction with alkyl amines to yield the grafting of the hydrophobic alkyl moiety via an amide linkage, with the concomitant formation of a carboxylic group. A differential feature of this approach is that the hydrophobicity of the resulting material is a balance between the ionization of the carboxylic groups and the hydrophobicity of the grafted amines.

The solvent free thermolysis of CA yields AA-CDs as a solid material stuck to the flask that forces the use of a solvent for the reaction with the alkyl amine. To avoid this manipulation we hypothesized that if the solvent, in addition to the inertness toward the anhydride groups and the good miscibility/solubility with the hydrophobic amine, presents thermal stability, the two-step synthesis can be conducted in the same pot. DMSO, with a boiling point of $189^{\circ} \mathrm{C}$ at $1 \mathrm{~atm}$ and vapor pressure of $0.6 \mathrm{~mm}$ at $25{ }^{\circ} \mathrm{C}$ was the preferred solvent. This choice is further supported because DMSO is a green solvent, ${ }^{38,39}$ well ranked in different selection guides ${ }^{40,41}$ and it has been already used in the synthesis of CDs. ${ }^{42,43}$

\section{Thermolysis of CA in DMSO}

The appropriateness of the proposed design was evaluated by heating CA at $180{ }^{\circ} \mathrm{C}$ for two hours using DMSO as a solvent. The FTIR spectrum of the resulting liquid sample, named CD-1, (Fig. 1A) showed that the signals of the $\mathrm{O}-\mathrm{H}$ stretching of the
$\mathrm{CA}$ at 3500 and $3285 \mathrm{~cm}^{-1}$ vanished and those corresponding to the $\mathrm{C}=\mathrm{C}$ stretching $\left(1650 \mathrm{~cm}^{-1}\right)$ and bending $\left(880-1014 \mathrm{~cm}^{-1}\right)$ appeared, in full agreement with the transformation of CA into CDs. The analysis also revealed a double signal at 1844 and $1766 \mathrm{~cm}^{-1}$ that is the characteristic spectral signature of the asymmetric and symmetric $\mathrm{C}=\mathrm{O}$ stretching of acid anhydrides, supporting the use of DMSO as a solvent to yield AA-CDs. CD-1, was treated with a $\mathrm{NaOH}$ solution to hydrolyze the anhydride groups, dialyzed against water and lyophilized to yield CD-1Na. The FTIR spectrum of CD-1Na (Fig. 1A) did not show the double signal at 1844 and $1766 \mathrm{~cm}^{-1}$ but those of the $\mathrm{O}-\mathrm{H}$ stretching and the typical symmetric stretching vibration signature of carboxylic acid salts separated by $150 \mathrm{~cm}^{-1}$ and peaks at $1550 \mathrm{~cm}^{-1}$ and $1400 \mathrm{~cm}^{-1}$, further confirming that the thermolysis of CA in DMSO yields acid anhydride coated CDs. ${ }^{44}$

The surface chemistry of CD-1Na was characterized by XPS. The full scan XPS spectrum (Fig. S1A, ESI $\dagger$ ) showed peaks at ca. $285 \mathrm{eV}, 532 \mathrm{eV}, 1071 \mathrm{eV}$ and $164 \mathrm{eV}$, indicating that the sample contains $\mathrm{C}, \mathrm{O}, \mathrm{Na}$ and sulfur. The content of sulfur was estimated to be $0.3 \%$ (Table $\mathrm{S} 1, \mathrm{ESI} \dagger$ ) and it was initially assigned to residual DMSO. The high resolution $\mathrm{C}$ 1s spectrum was fitted with three peaks centred at $248.4 \mathrm{eV}, 286.2 \mathrm{eV}$ and $288.1 \mathrm{eV}$ which are assigned to graphitic carbon, $\mathrm{C}-\mathrm{O}$ and $\mathrm{C}=\mathrm{O}$, respectively (Fig. S1B, ESI $\dagger$ ). ${ }^{45}$ The $\mathrm{O}$ 1s spectrum (Fig. S1C, ESI $\dagger$ ), besides the peaks centred at $530.9 \mathrm{eV}$ and $531.3 \mathrm{eV}$ corresponding to $\mathrm{O}=\mathrm{C}$ and $\mathrm{O}-\mathrm{C}$, consisted of a third peak at $535.4 \mathrm{eV}$ attributed to Na KLL Auger emission. ${ }^{46}$ The analysis of the high resolution $\mathrm{S} 2$ p spectrum(Fig. 1B) was fitted with three peaks centred at $163.3 \mathrm{eV}, 164.5$ and $165.9 \mathrm{eV}$, the latter being the only coincident with the features of the $S 2 p$ spectrum of DMSO. ${ }^{47}$

The position of the two former peaks, the spacing of $1.18 \mathrm{eV}$ and the intensity ratio of 0.48 agree with the $2 \mathrm{p}_{3 / 2}$ and $2 \mathrm{p}_{1 / 2}$ spin-orbit splitting of the $-\mathrm{C}-\mathrm{S}-$ covalent bond of the thiophene-S. $^{48-51}$ This result discards a contamination with DMSO and demonstrates that the thermolysis of CA in DMSO yields the incorporation of sulfur to the CDs.

Sulfur is an interesting dopant because it shares with carbon similar electronegativity (2.55 for carbon and 2.58 for sulfur) but different size (empirical atomic radius of $70 \mathrm{pm}$ for carbon and $100 \mathrm{pm}$ for sulfur), features that make feasible the incorporation of sulfur to aromatic rings and conjugation to planar ring carbon frameworks. ${ }^{52}$ Indeed, sulfur doping has been reported to improve quantum yield and shift the wavelength the maximum emission peak. ${ }^{53-55}$ Hence, morphology, size and photoluminiscence of CD-1Na were investigated. High-resolution transmission electron microscopy (HR-TEM) revealed spherical particles with an average diameter of $2.2 \mathrm{~nm}$ and selected area electron diffraction pattern supported the crystalline nature of CD-1Na with an interplanar spacing of $0.275 \mathrm{~nm}$ (Fig. S2A, ESI $\dagger$ ). The emission spectrum of CD-1Na was very similar to that for the CDots obtained by solvent free thermolysis of $\mathrm{CA}^{37}$ sharing the excitation at $326 \mathrm{~nm}$ and emission at $454 \mathrm{~nm}$ peaks (Fig. S2B, ESI $\dagger$ ), and a quantum yield of $4 \%$ (Table S2, ESI $\dagger$ ). A closer analysis of the emission spectrum of CD-1Na (Fig. S2B, ESI $\dagger$ ) revealed a shoulder at $435 \mathrm{~nm}$ that can be rationalized 

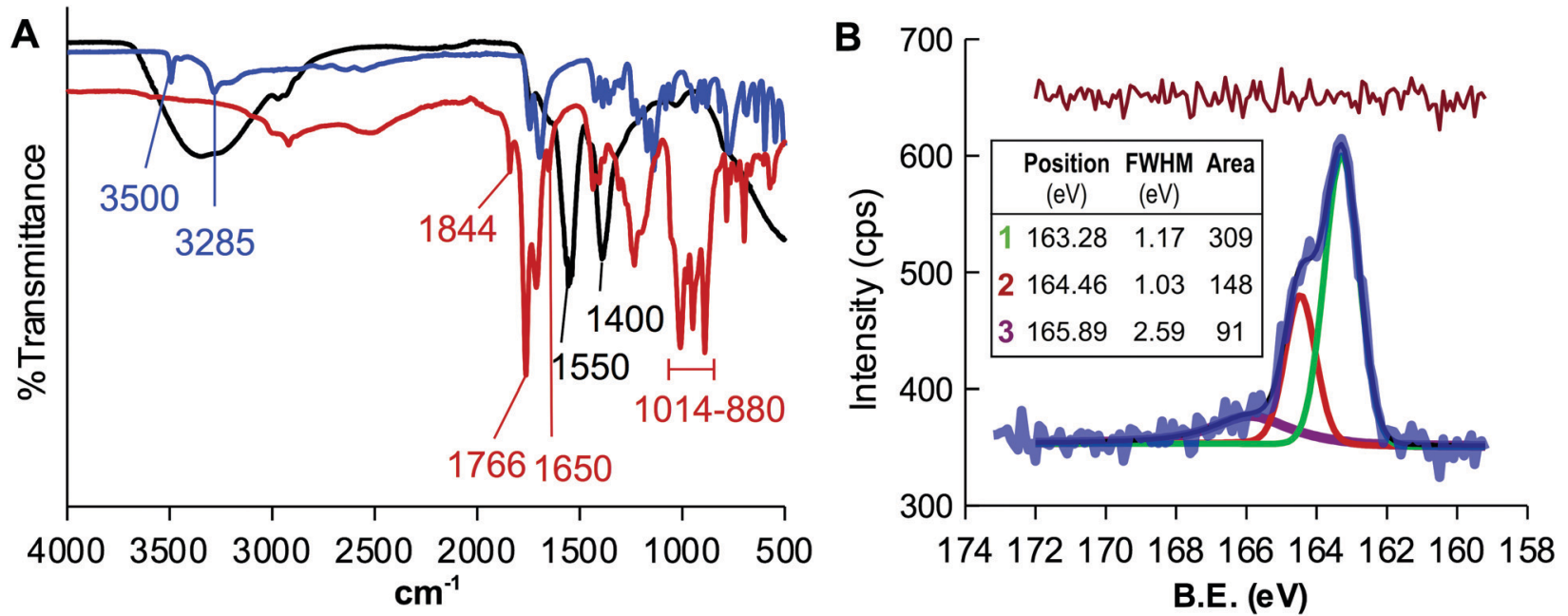

Fig. 1 (A) FTIR spectrum of CA (blue), of the solution resulting from the thermolysis of CA in DMSO (red), and after treatment with a $\mathrm{NaOH}$, dialysis and lyophilization (black). (B) S 2p high resolution XPS spectrum of CD-1Na. Experimental data are shown in light blue, envelope in black and residuals on top in brown. Inset, table showing the main features of the peaks resulting from the peak fitting.

considering that the thermolysis of $\mathrm{CA}$ is a complex process that involves the formation of several intermediates to yield a combination of CDs with different emission peaks. In particular, the intermediates trans-aconitic acid and itaconic acid have been reported to yield fluorescent CDs that emit at lower wave-lengths and that the relative abundance of the different intermediates is influenced by reaction conditions. ${ }^{56}$

\section{Functionalization of CD-1 with alkyl chains}

The presence of acid anhydride groups in CD-1 makes them reactive and functionalizable without any previous step of activation. ${ }^{37}$ Our attention was focused on exploiting this feature to attach alkyl chains as hydrophobic moiety. Hexane1-amine and octadecan-1-amine were selected on the basis that they share reactivity toward CD-1 but differ in their contribution to hydrophobicity, the octanol water partition coefficient in terms of $\log$ P being estimated as 1.63 and 5.37 respectively. ${ }^{57}$

Thus, the thermolysis of CA in DMSO and, without purification, later reaction with hexane-1-amine or octadecan-1-amine yielded products LCD-1 and LCD-2, respectively. LCDs were purified by dilution of the crude reaction mixture with toluenediethyl ether and washing with $5 \% \mathrm{HCl}$ to isolate the LCDs from the organic phase. XPS analysis found $0.8 \%$ and $1.1 \%$ of $\mathrm{N}$ for LCD-1 and LCD-2 (Table S1, ESI $\dagger$ ), confirming the alkylation of CD-1. The deconvolution of the $\mathrm{N}$ 1s peak revealed the expected signal at $399.8 \mathrm{eV}$ for the amide-type nitrogen and a second signal at $401.6 \mathrm{eV}$ that was assigned to amine-type nitrogen (Fig. 2A and B). ${ }^{58}$ However, the ratio between both peaks was close to 1 , pointing to the presence of alkyl amine in spite of the extraction with $5 \% \mathrm{HCl}$.

In order to assess the elimination the alkyl amine, the organic phases containing LCDs were further purified by washing with a solution of sodium bicarbonate. The luminescence was observed to migrate to the aqueous phase due to the deprotonation of the carboxylic groups to form the sodium salts LCD-1Na and LCD-2Na. After exhaustive dialysis against water followed by lyophilization, LCD-1Na and LCD-2Na were characterized by XPS and HRTEM. The deconvolution of the $\mathrm{N} 1 \mathrm{~s}$ peak showed that the signal assigned to unreacted alkyl amine persisted and the amide:amine ratio remained close to 1 for LCD-2Na (Fig. 2D) but increased to 6 for LCD-1Na (Fig. 2C). These results suggest that the reaction of CD-1 with the alkyl amines yields the grafting of the alkyl chain by both covalent bonds (i.e. amide-type) and non-covalent interactions, the latter being dependent on the length of the alkyl chain. Thus, whereas for short chain alkyl amines the non-covalent interactions are mainly ionic and disrupted by $\mathrm{pH}$, for long alkyl amines they are mainly hydrophobic, due to interactions among the alkyl chains, and insensitive to $\mathrm{pH}$. At this point it is important to highlight that the unreacted amines present in LCD-2 and LCD-2Na are part of the CDs and do not exchange with the solvent. HRTEM analysis revealed that LCDs are spherical nanoparticles with an average diameter of $2.5 \mathrm{~nm}$, with 0.14 as the coefficient of variation (Fig. 3A). As expected, they are fluorescent and their spectra show a $20 \mathrm{~nm}$ blue shift of the emission peak relative to CD-1Na (Fig. 3B). The quantum yield of LCD-1Na is similar to that of CDN-1, but larger than that of LCD-2Na (Table S2, ESI $\dagger$ ), pointing to a lower fluorescent mass:overall mass ratio, in agreement with the longer alkyl chains appended to LCD-2Na.

The fact that LCDs can be transferred from the organic phase to the aqueous phase and vice versa resembles amphibious ${ }^{17}$ or amphiphilic ${ }^{15,24,26}$ CDs. In order to gain additional insight into the features of LCDs, the Z-potential (Table S3, ESI $\dagger$ ) and the octanol water partition coefficient $(\log P)$ (Table 1$)$ were estimated. The values of $\log P$ are within the $-0.5<\log P<5$ range predicted by the Lipinski rule of five for compounds with suitable cell permeability. Salt forms are highly hydrosoluble, LCD-2Na being the least hydrosoluble, and the lipophilicity can be modulated by the protonation/deprotonation of the carboxylate groups. As expected from previous studies that have demonstrated that 

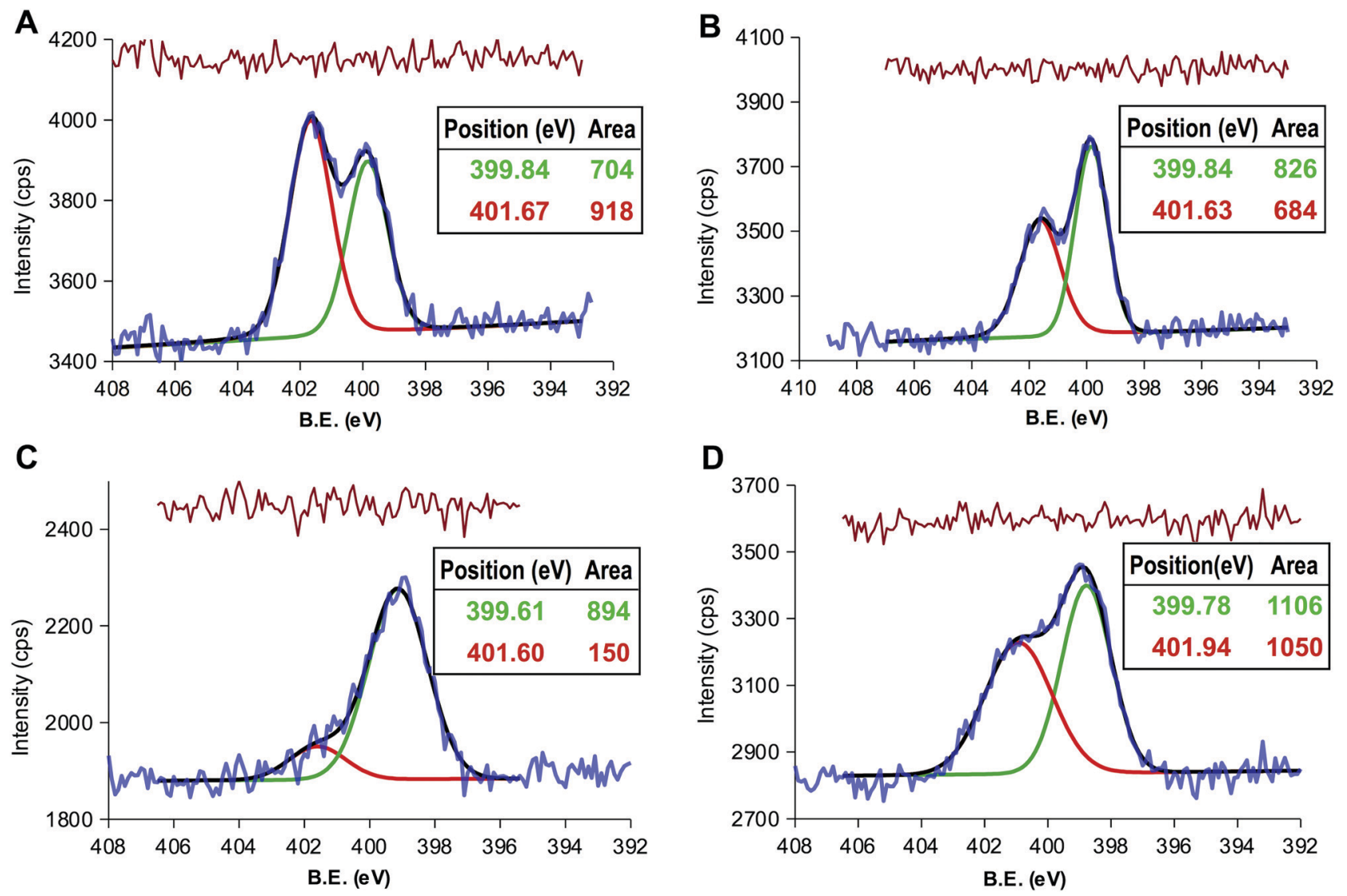

Fig. $2 \mathrm{~N}$ 1s high resolution XPS spectra of LCD-1 (A) LCD-2 (B) LCD-1Na (C) and LCD-2Na (D) experimental data are shown in light blue, envelope in black and residuals on top in brown. Inset, table showing the main features of the peaks resulting from the peak fitting.

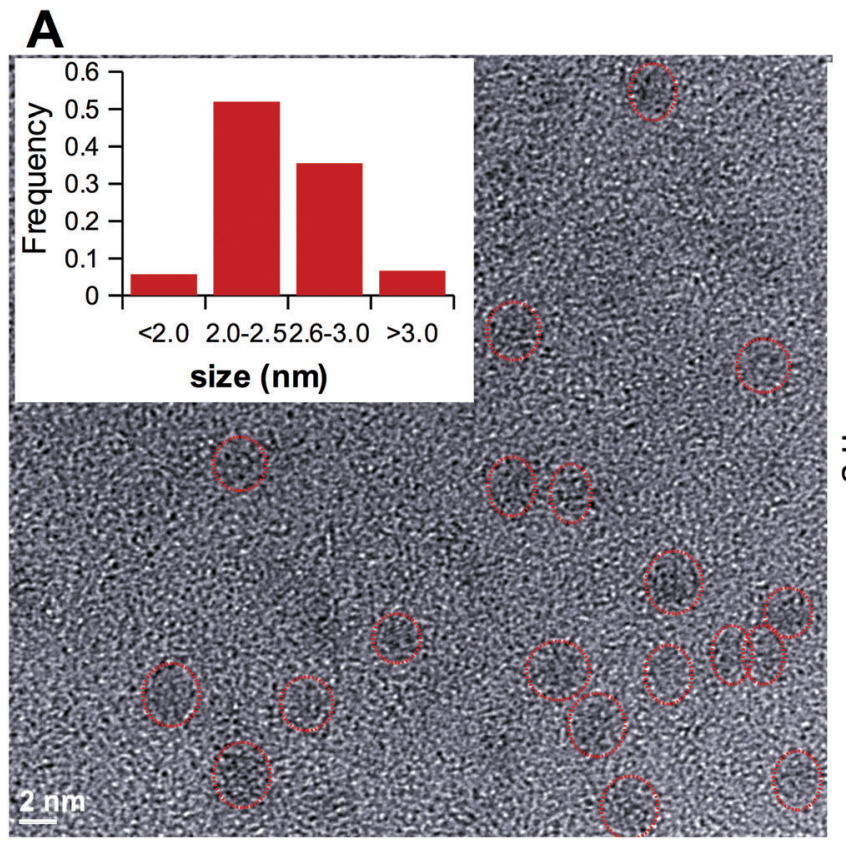

B

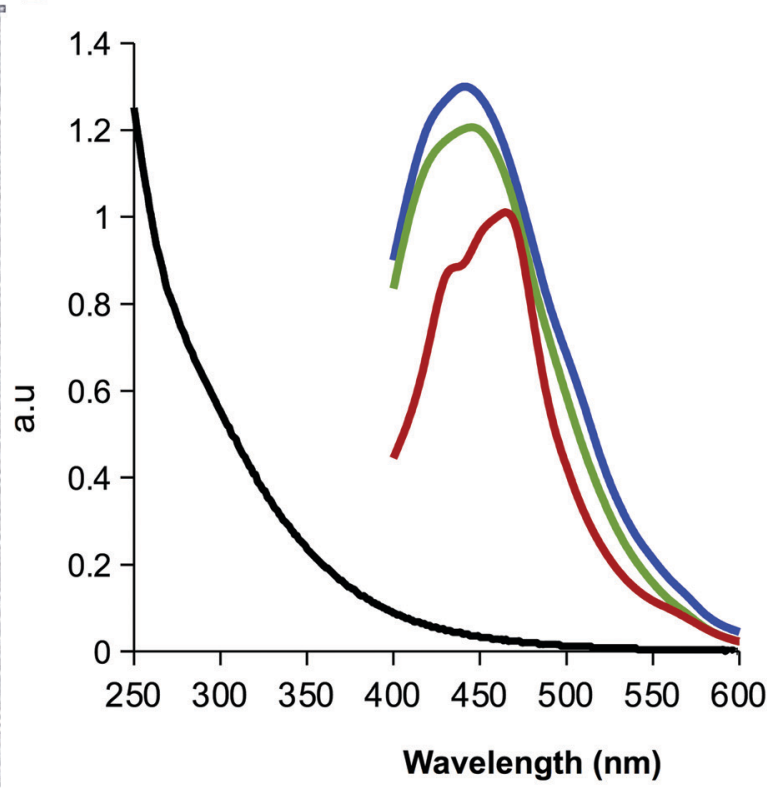

Fig. 3 (A) HRTEM image of LCND-2Na showing the size distribution (inset). (B) UV-Vis absorption (black line) and photoluminescence emission (excitation at $326 \mathrm{~nm}$ ) spectra of LCD-1Na (green), LCD-2Na (blue) and CD-1Na (red). 
Table 1 Octanol-water partition coefficient of both the sodium salt and the acidic form of CDs obtained by solvent free thermolysis (AA-CDNa and AA-CD), in DMSO (CD-1Na, CD-1,LCD-1Na, LCD-1, LCD-2Na and LCD-2)

\begin{tabular}{llll}
\hline Sample & $\log P$ & Sample & $\log P$ \\
\hline AA-CDNa & $-{ }^{a}$ & AA-CD & 0.09 \\
CD-1Na & $-{ }^{a}$ & CD-1 & 0.79 \\
LCD-1Na & $-{ }^{a}$ & LCD-1 & 1.03 \\
LCD-2Na & -1.05 & LCD-2 & 0.89 \\
${ }^{a}$ Particles remain in the aqueous phase. & &
\end{tabular}

sulfur increases the hydrophobicity of nanoscale zero-valent iron, the incorporation of sulfur shifts the $\log P$ from 0.09 for AA-CDs obtained by solvent free thermolysis of CA to 0.79 for CD-1. ${ }^{59}$ When the C18 alkyl chains are grafted, the $\log P$ increases to 1.03, which is slightly above the threshold value $(\log P \geq 1)$ to distinguish membrane permeant from impermeant compounds. ${ }^{60}$ These values support the amphiphilic nature of LCDs and their ability to transverse membranes.

\section{Zeta potential of the CDs}

The effect of the coating structure of the CDs and of $\mathrm{pH}$ on the zeta potential of the carbon dots is a significant feature of these nanoparticles, considering their potential application as drug vehicles to be injected in the body and subjected to environments of either physiological (7.4) or tumoral (6.5) pH values. The pertinent data are presented in Table S3 (ESI $\dagger$ ), and they show that the modification of $\mathrm{pH}$ in the interval mentioned does not affect beyond experimental uncertainty the zeta potential.

\section{Application of LCDs}

Since LCDs consist of a carbon core bearing ionizable carboxylic groups and hydrophobic flexible alkyl chains that can establish hydrophobic interactions, we hypothesize that LCDs can act as supramolecular hosts of hydrophobic guest molecules. With this feature LCDs can behave as nanocarriers in a manner that resembles other supramolecular hosts, but with additional advantages since the size of the hosted guest can be modulated by the length of the alkyl chains and the solubility of the complex by pH. Additionally, the cytotoxicity assay of LCDs against the cell line HEK 239 revealed that they are well tolerated (Fig. S3, ESI $\dagger$ ), anticipating their potential in biomedical applications, as their hydrosoluble counterparts. ${ }^{61}$

The hypothesis was put to test with the hydrophobic drug camptothecin (CPT) and with the NIR fluorescent hydrophobic dye IR780. The former is a potent chemotherapeutic agent ${ }^{27}$ and the latter, besides the emission in the $807-823 \mathrm{~nm}$ wavelength range that makes it suitable for bioimaging, shows an absorption peak at $792 \mathrm{~nm}$ that yields a temperature increase and a production of ROS upon illumination with NIR light, enabling its use in photothermal and photodynamic therapies. ${ }^{29}$ However, the clinical implementation of both molecules is limited by their poor solubility, the improvement of which being a challenging task.

\section{LCDs as antitumor drug vehicles}

From a structural point of view the planar structure of CPT is expected to favour the intercalation in the alkyl chains. The formation of the LCD@CPT inclusion complexes was approached by combining an aqueous solution of the sodium salt of LCDs in water and CPT in the organic phase and either removing the organic phase to force the formation of inclusion complexes or shaking overnight. Regardless the procedure, LCD-1Na did not form inclusion complexes with CPT. This result was not unexpected considering that the shorter length of the alkyl chain of the hexane-1-amine compared to the size of CPT may be insufficient to form stable inclusion complexes (Fig. S4, ESI $\dagger$ ). LCD-2Na forms stable complexes by both approaches, but the load of CPT was 3-fold higher when the formation of the inclusion complexes proceeded by shaking overnight $\left(253 \pm 13 \mu \mathrm{g} \mathrm{mg}^{-1}\right.$ vs. $\left.85 \pm 25 \mu \mathrm{g} \mathrm{mg}^{-1}\right)$. When LCD2Na is loaded with CPT to yield LCD-2Na@CPT the effect on the viability of the HEK 239 is in the range and even slightly better than an equivalent amount of free fresh CPT (Fig. S5, ESI $\dagger$ ). This result is encouraging when recalling that CPT from LCD2Na@CPT underwent a processing incompatible with the stability of free CPT. Additionally, it confirms that the interaction between LCD-2Na and CPT is reversible and that the released drug is functional. In order to further evaluate the application of LCD-2Na as carrier of CPT, the cytotoxicity of LCD-2Na@CPT was assessed against a battery of cell lines and compared with an equivalent amount of free fresh CPT (Fig. 4). As depicted in Table 2 the IC50 of free CPT is cell line dependent and when compared to LCD-2Na@CPT it is in the same range for N2a, CT26wt and SKBR3. This result is remarkable when considering that it is assumed for the comparison that the full load of CTP is active and released from LCD-2Na@CPT. For the cell lines G361 and HeLa the IC50 of LCD-2Na@CPT is two- and four-fold higher than that for free CPT, suggesting an influence of the carrier. Flow cytometry analysis on the cell line G361 confirmed the result (Fig. S6, ESI $\dagger$ ). Overall, these data support the potential of LCD-2Na as a drug carrier.

\section{LCDs in photothermal therapy}

The formation of the inclusion complexes with IR780 is more challenging since the molecule is not planar. Based on the structure of IR780 and the length of the alkyl chains of LCDs (Fig. S4, ESI $\dagger$ ) LCD-1(Na) were discarded and the formation of inclusion complexes with LCD-2(Na) were approached by two strategies: (i) combination of the hydrosoluble form of LCD (i.e. LCD-2Na) in water and IR780 in the organic phase, or alternatively (ii) both, LCD-2 and IR780 in the organic phase. Since CDs absorb in the UV-region and IR780 shows a characteristic peak at $792 \mathrm{~nm}$, the absorption spectra allow the estimation of the IR780 load. Data reveal that the latter strategy was the most successful in the formation of the inclusion complexes (Fig. S7, ESI $\dagger$ ).

As a proof of concept to evaluate use of LCD-2Na@IR780 in photothermal therapy, the thermal behaviour of a solution of the latter containing $72 \mu \mathrm{M}$ of IR780 was compared with a 
HeLa (cervical carcinoma)

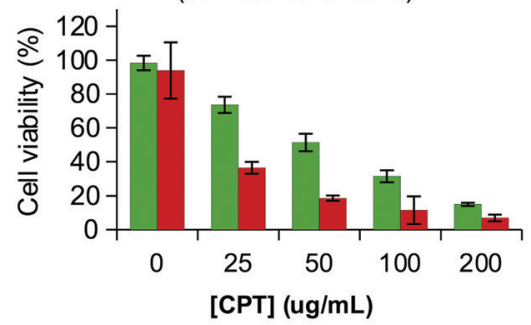

CT26wt

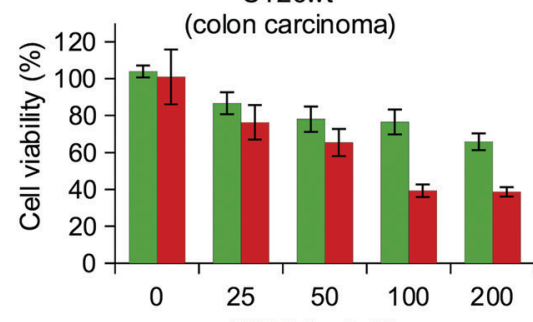

[CPT] $(\mu \mathrm{g} / \mathrm{mL})$
G361

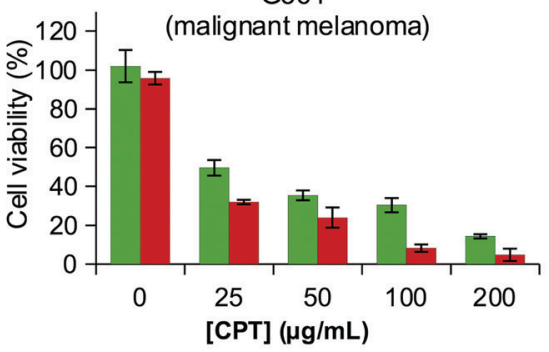

SKBR3

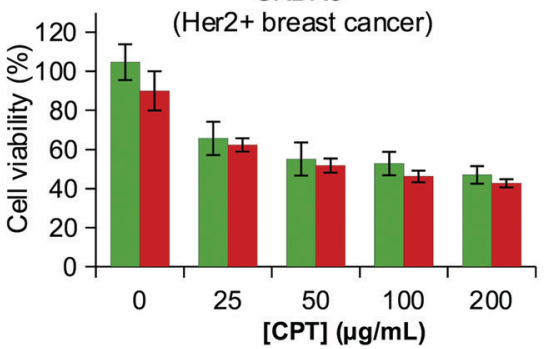

N2a

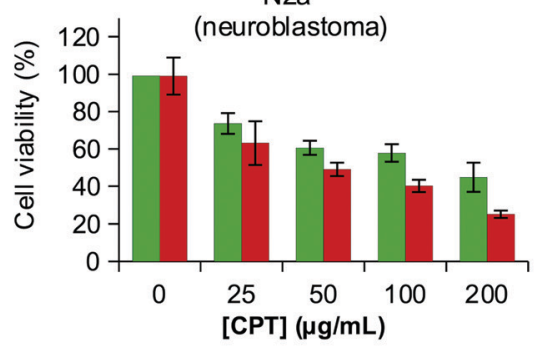

MDA-MB-231

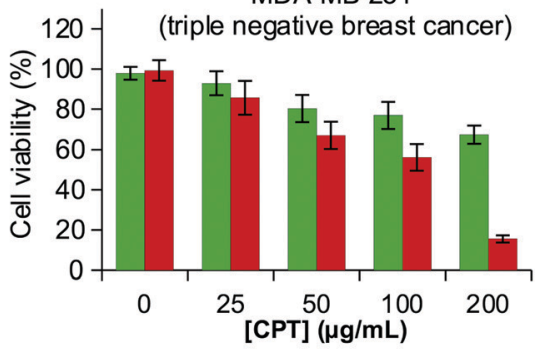

Fig. 4 Comparison of the cytotoxicity of LCD-2Na@CPT (green) and free CPT (red) on different cancer cell lines at different equivalent concentrations of CPT. Cell lines were incubated for $24 \mathrm{~h}$ with suitable amounts of either free CPT or LCD-2Na@CPT and cytotoxicity was assayed by the MTT method. Results are means \pm 1 standard deviation.

Table 2 IC50 values (in $\mu$ M) of free CPT and LCD-2Na@CPT against a panel of cancer cell lines

\begin{tabular}{lll}
\hline Cell line & Free CPT & LCD-2Na@CPT \\
\hline HeLa & 46 & 189 \\
G361 & 58 & 114 \\
SKBR3 & 115 & 139 \\
N2a & 170 & 160 \\
CT26wt & 213 & 215 \\
MDA-MB-231 & 264 & $>575^{a}$
\end{tabular}

${ }^{a}$ Highest concentration assayed due to solubility limitations.
$133 \mu \mathrm{M}$ solution of the closely related hydrosoluble hepatamethyne IR783. As shown in Fig. 5A, upon irradiation with a $808 \mathrm{~nm}$ laser $\left(1.4 \mathrm{~W} \mathrm{~cm}^{-2}\right)$ for 5 minutes, both solutions showed similar behaviour during the first 66 seconds, with a heating rate of $0.3{ }^{\circ} \mathrm{C}$ per second. However, whereas the temperature of the LCD-2Na@IR780 solution continued increasing to rise up to $50{ }^{\circ} \mathrm{C}$ after 135 seconds and maintained the temperature for about $1 \mathrm{~min}$, the temperature of the solution with IR783 declined abruptly after reaching $44.6{ }^{\circ} \mathrm{C}$. To gain additional insight into this differential behaviour, drops containing a diluted solution of IR783 (i.e. $13 \mathrm{nM}$ ) were illuminated with the laser for 30 seconds. Thermal images showed that within this short period of time the drop behaved
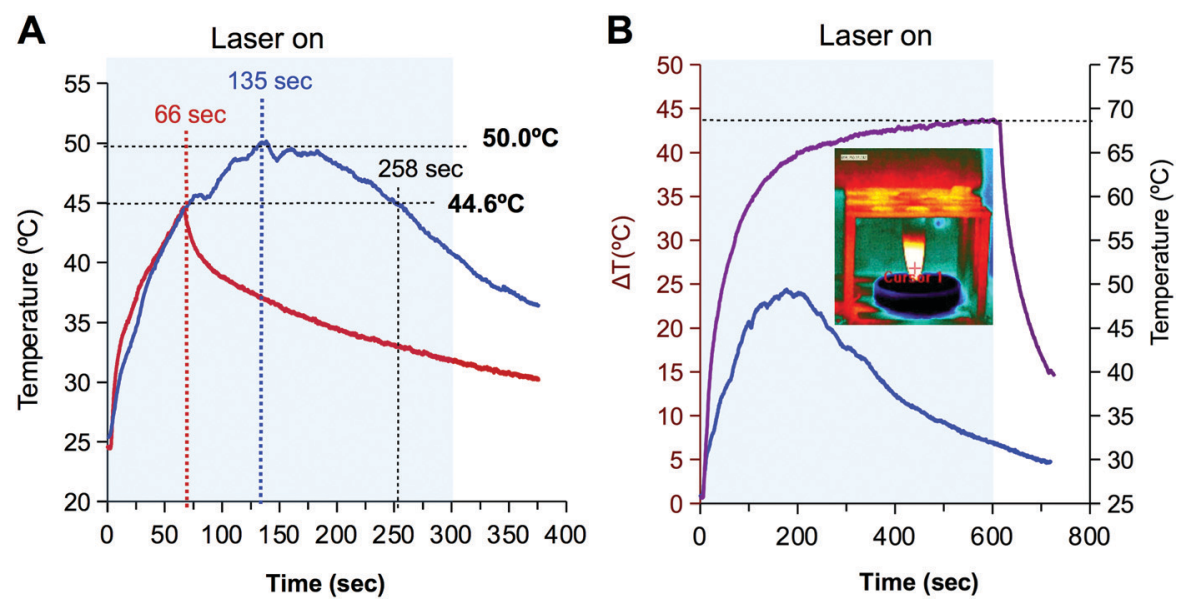

Fig. 5 Temperature increase as a function of time of $200 \mu \mathrm{L}$ of a water solutions containing $133 \mu \mathrm{M}$ IR783 (red), $72 \mu \mathrm{M} \mathrm{LCD-2Na@IR780}$ (blue) and $285 \mu$ M LCD-2Na@IR780 (magenta) irradiated with a $808 \mathrm{~nm}$ NIR laser at a power of $1.2 \mathrm{~W} \mathrm{~cm}^{-2}$ for $5 \mathrm{~min}$ (A) or 10 min (B). Inset B: Thermal image of the epperdorf containing the LCD-2Na@IR780 solution and the sample holder during the illumination. The temperature was recorded in real time with a high-resolution infrared camera. 
as the $72 \mu \mathrm{M}$ solution, with an increase of temperature for the first seconds and then cooling down (Fig. S8, ESI $\dagger$ ). Visual inspection of the drop showed that its colour changed from the characteristic blue to yellowish and the absorption spectrum revealed that the peak at $780 \mathrm{~nm}$ vanished. These observations have been ascribed by other authors to the generation of singlet oxygen and degradation of IR780 into dioxetanes and carbonyl compounds. ${ }^{30,62}$

The above results suggest that the thermal profile is dependent on the concentration of NIR dye and that the formation of a complex with LCD-2 spans its life. This hypothesis was confirmed when the thermal profiles of two solutions of LCD-2Na@IR780 containing $72 \mu \mathrm{M}$ and $258 \mu \mathrm{M}$ of IR780 were compared. As shown in Fig. $5 \mathrm{~B}$, the $72 \mu \mathrm{M}$ solution behaved as expected from the previous experiment, reaching a maximum temperature after $3 \mathrm{~min}$ and then cooling down due to the destruction of the dye. However the $258 \mu \mathrm{M}$ solution withstood 10 min of irradiation with the laser and the temperature reached $68.5{ }^{\circ} \mathrm{C}$, with a slope of $19{ }^{\circ} \mathrm{C}$ in the first 30 seconds that flattened to $2{ }^{\circ} \mathrm{C}$ in the last $5 \mathrm{~min}$ of irradiation, and the temperature did not drop until the laser was turned off. These results lead to conclude that the irradiation with the laser yields the formation of oxygen singlet with the concomitant destruction of IR780 and that the concentration determines not only the maximum temperature reached but also the time that the laser is effective. At this point, it is important to recall that temperatures above $48{ }^{\circ} \mathrm{C}$ for minutes provoke irreversible injury that is enhanced by the reactive species produced during the destruction of IR780 by the laser. ${ }^{29}$

\section{Experimental}

\section{Thermolysis of CA}

Thermolysis of CA was carried out by modification of the protocol previously reported for AA-CDs. ${ }^{37}$ Briefly, an amount of $10 \mathrm{~g}$ of CA was placed in an open-round bottom flask and heated in an oil bath at $180{ }^{\circ} \mathrm{C}$ for $2 \mathrm{~h}$ in $10 \mathrm{~mL}$ of anhydrous DMSO to yield CD-1.

\section{Synthesis of $\operatorname{LCD}(\mathrm{Na})$}

In the same open-round bottom flask where $\mathbf{C D}-\mathbf{1}$ were obtained by thermolysis at $180{ }^{\circ} \mathrm{C}$ of $10 \mathrm{~g}$ of CA in $10 \mathrm{~mL}$ of anhydrous DMSO, $1 \mathrm{~mL}$ of the solution was reacted overnight at room temperature with $0.5 \mathrm{mmol}$ of, either hexane-1-amine $(70 \mu \mathrm{L})$ or octadecan-1-amine $(140 \mathrm{mg})$ in the presence of $0.5 \mathrm{mmol}$ of triethylamine $(70 \mu \mathrm{L})$ to yield LCD-1 and LCD-2 respectively. For the isolation, a volume of $20 \mathrm{~mL}$ of ether: toluene $(1: 1)$ was added and then washed with $5 \% \mathrm{HCl}(20 \mathrm{~mL})$. The organic phase was collected, treated with anhydrous $\mathrm{Na}_{2} \mathrm{SO}_{4}$ and the solvent eliminated with a rotary evaporator to yield CDs decorated with hexane-1-amine (LCD-1, 0.859 g) or with octadecan-1-amine (LCD-2, 0.441 g). Sodium salts (LCD-1Na and LCD-2Na) were obtained by extraction of LCDs with a saturated solution of $\mathrm{HCO}_{3} \mathrm{Na}$, exhaustive dialysis (MWCO $=14000 \mathrm{Da}$ ) against deionized water and then lyophilization.

\section{Preparation of LCD-2Na@CPT for biological assays}

A volume of $14 \mathrm{~mL}$ of a $0.5 \mathrm{mg} \mathrm{mL} \mathrm{mL}^{-1}$ solution of LCD-2Na in water was combined with $14 \mathrm{~mL}$ of $0.5 \mathrm{mg} \mathrm{mL}^{-1}$ solution of CPT in $\mathrm{Cl}_{3} \mathrm{CH}$. The mixture was shaken at room temperature and protected from light for $24 \mathrm{~h}$ and phase separation was allowed to proceed for 48 hours. The organic R3 phase was discarded and the concentration of CPT in the aqueous phase was estimated from the remaining fluorescence in the discarded organic phase.

\section{Preparation of LCD-2@IR780 for photothermal assay}

A volume of $6 \mathrm{~mL}$ of a $0.5 \mathrm{mg} \mathrm{mL} \mathrm{m}^{-1}$ solution of IR780 in $\mathrm{Cl}_{3} \mathrm{CH}$ was combined with $6 \mathrm{~mL}$ of LCD-2 in $\mathrm{Cl}_{3} \mathrm{CH}\left(0.5 \mathrm{mg} \mathrm{mL}{ }^{-1}\right)$. After sonication for $2 \mathrm{~min}$, it was shaken overnight at room temperature protected from light. The sample was extracted with $50 \mathrm{mM}$ carbonate-bicarbonate buffer pH $9(3 \times 4 \mathrm{~mL})$. The concentration was estimated from the interpolation in the suitable calibration curves of absorbance at $326 \mathrm{~nm}$ and fluorescence at $454 \mathrm{~nm}$ (Ex $326 \mathrm{~nm}$ ) for LCDs and of absorbance at $792 \mathrm{~nm}$ for IR780.

\section{Cytotoxicity of LCD-2Na@CPT}

Cell lines were incubated for $24 \mathrm{~h}$ with suitable amounts of either free CPT or LCD-2Na@CPT to yield final CPT concentrations up to $200 \mu \mathrm{g} \mathrm{mL}{ }^{-1}$. Cytotoxicity was assayed by determining the percentage of cell viability (with respect to unexposed cells) using the 3-(4,5-dimethylthiazol-2-yl)-2,5-diphenyl-2 $\mathrm{H}^{-}$ tetrazolium bromide (MTT) method. Results are reported as $\%$ viability based on the untreated control cells at $24 \mathrm{~h}$ normalized to $100 \%$ viable. ED50plus v1.0 software was used to calculate IC50.

\section{Photothermal measurements}

Water solution samples of $200 \mu \mathrm{L}$ contained in $1.5 \mathrm{~mL}$ Eppendorf tubes were irradiated with a near-infrared continuous laser at a wavelength of $808 \mathrm{~nm}$ for $10 \mathrm{~min}$. The laser power was calibrated at $360 \mathrm{~mW}$, corresponding to a power density of $1.2 \mathrm{~W} \mathrm{~cm}^{-2}$. The distance between the sample and the laser was $6 \mathrm{~cm}$, and the laser spot in the sample was $0.3 \mathrm{~cm}^{2}$. The temperature profile as a function of time was recorded in real time with a high-resolution infrared camera FLIR E60 with $320 \times 240$ pixels IR resolution and thermal sensitivity better than $0.05{ }^{\circ} \mathrm{C}$ (FLIR Systems Inc., USA). Data was processed with FLIR RESEARCHIR MAX 4 thermal analysis software. (provided by the manufacturer).

\section{Conclusions}

The one-pot thermolysis of CA in DMSO and further reaction with alkyl amines is a suitable approach to obtain amphiphiliclike CDs bearing hydrophobic alkyl chains and carboxylic groups. The alkyl chains allow the formation of inclusion complexes with hydrophobic guest whereas the ionization of the carboxylic groups can be modulated by $\mathrm{pH}$, determining the hydrosolubility of the complexes. LCDs are well tolerated by 
cells and, using a suitable length of the alkyl chains, they form inclusion complexes with hydrophobic guests of complementary size. The values of $\log P$ and the results obtained from the model drug CPT support the biotechnological potential of LCDs as drug carriers that overcome solubility problems and protect from degradation. The IC50 of LCD-2Na@CPT is in the same range as that of free IC50 for the cancer cell lines $\mathrm{N} 2 \mathrm{a}$ (neuroblastoma), CT26wt (colon carcinoma), SKBR3 (Her2+ breast cancer), twofold higher for G361 (malignant melanoma) and fourfold higher for HeLa (cervical carcinoma). The performance of LCD-2Na complexes with IR780 dye, yielding a significant sustained increase of temperature of up to $68.5{ }^{\circ} \mathrm{C}$, also supports their application in photothermal therapy.

\section{Author contributions}

Conceptualization, M. O.-M., G. R. I., R. S. G., F. J. L.-J., F. S.-G.; formal analysis, F. J. L.-J., M. O.-M; funding acquisition, M. D. G.-G., A. V. D., F. S. G.; investigation, M. O.-M., P. V.-N., S. P., M. D. G.-G., G. R. I., F. J. L.-J.; methodology, M. O.-M, G. R. I., F. J. L.-J.; M. D. G.-G.; supervision, R. S.-G., A. V. D., F. S.-G.; validation, G. R. I., F. J. L-J., visualization, G. R. I., M. D. G.-G., F. H. M., F. J. L.-J; writing - original draft, F. J. L.-J.; writing review \& editing: G. R. I., M. D. G.-D., R. S.-G., F. H.-M., A. V., F. J. L.-J., F. S.-G. All authors have read and agreed to the published version of the manuscript.

\section{Conflicts of interest}

There are no conflicts to declare.

\section{Acknowledgements}

The work was financially supported by the Spanish institutions Ministerio de Ciencia, Innovación y Universidades (PGC2018098770-B-I00 and CTQ2017-86125-P) and Junta de Andalucía (ProgramaOperativo FEDER 2014-2020, grants B-FQM-141UGR18, A1-FQM-341-UGR-18, C-FQM-497-UGR18).

\section{References}

1 S. Sharma, A. Umar, S. Sood, S. K. Mehta and S. K. Kansal, Photoluminescent C-dots: an overview on the recent development in the synthesis, physiochemical properties and potential applications, J. Alloys Compd., 2018, 748, 818, DOI: 10.1016/j.jallcom.2018.03.001.

2 M. Semeniuk, Z. Yi, V. Poursorkhabi, J. Tjong, S. Jaffer and Z.-H. Lu, et al., Future Perspectives and Review on Organic Carbon Dots in Electronic Applications, ACS Nano, 2019, 13, 6224, DOI: 10.1021/acsnano.9b00688.

3 M. L. Liu, B. B. Chen, C. M. Li and C. Z. Huang, Carbon dots: synthesis, formation mechanism, fluorescence origin and sensing applications, Green Chem., 2019, 21, 449, DOI: 10.1039/C8GC02736F.
4 K. Ghosal and A. Ghosh, Carbon dots: the next generation platform for biomedical applications, Mater. Sci. Eng., C, 2019, 96, 887, DOI: 10.1016/j.msec.2018.11.060.

5 B. Thangaraj, P. R. Solomon and S. Ranganathan, Synthesis of Carbon Quantum Dots with Special Reference to Biomass as a Source - A Review, Curr. Pharm. Des., 2019, 25, 1455, DOI: $10.2174 / 1381612825666190618154518$.

6 N. Dhenadhayalan, K. Lin and T. A. Saleh, Recent Advances in Functionalized Carbon Dots toward the Design of Efficient Materials for Sensing and Catalysis Applications, Small, 2020, 16, 1905767, DOI: 10.1002/ smll.201905767.

7 F. Li, D. Yang and H. Xu, Non-Metal-Heteroatom-Doped Carbon Dots: Synthesis and Properties, Chem. - Eur. J., 2019, 25, 1165, DOI: 10.1002/chem.201802793.

8 L. Lin, Y. Luo, P. Tsai, J. Wang and X. Chen, Metal ions doped carbon quantum dots: synthesis, physicochemical properties, and their applications, TrAC, Trends Anal. Chem., 2018, 103, 87, DOI: 10.1016/j.trac.2018.03.015.

9 P. Zhao and L. Zhu, Dispersibility of carbon dots in aqueous and/or organic solvents, Chem. Commun., 2018, 54, 5401, DOI: 10.1039/C8CC02279H.

10 A. B. Bourlinos, A. Stassinopoulos, D. Anglos, R. Zboril, V. Georgakilas and E. P. Giannelis, Photoluminescent Carbogenic Dots, Chem. Mater., 2008, 20, 4539, DOI: 10.1021/ cm800506r.

11 Q.-X. Mao, W.-J. Wang, X. Hai, Y. Shu, X.-W. Chen and J.-H. Wang, The regulation of hydrophilicity and hydrophobicity of carbon dots via a one-pot approach, J. Mater. Chem. B, 2015, 3, 6013, DOI: 10.1039/C5TB00963D.

12 K. Yin, D. Lu, L. Wang, Q. Zhang, J. Hao and G. Li, et al., Hydrophobic Carbon Dots from Aliphatic Compounds with One Terminal Functional Group, J. Phys. Chem. C, 2019, 123, 22447, DOI: 10.1021/acs.jpcc.9b04479.

13 S. D. Çalhan, M. Ö. Alaş, M. Aşık, F. N. D. Kaya and R. Genç, One-pot synthesis of hydrophilic and hydrophobic fluorescent carbon dots using deep eutectic solvents as designer reaction media, J. Mater. Sci., 2018, 53, 15362, DOI: 10.1007/ s10853-018-2723-4.

14 Q.-X. Mao, E. Shuang, J.-M. Xia, R.-S. Song, Y. Shu and X.-W. Chen, et al., Hydrophobic Carbon Nanodots with Rapid Cell Penetrability and Tunable Photoluminescence Behavior for in Vitro and in Vivo Imaging, Langmuir, 2016, 32, 12221, DOI: 10.1021/acs.langmuir.6b03331.

15 S. Sarkar, S. Dinda, P. Choudhury and P. Kumar Das, Selfassembly of surface functionalized amphiphilic carbon dots: tuning in morphological manifestations, Soft Matter, 2019, 15, 2863, DOI: 10.1039/C9SM00051H.

16 M. Varisco, D. Zufferey, A. Ruggi, Y. Zhang, R. Erni and O. Mamula, Synthesis of hydrophilic and hydrophobic carbon quantum dots from waste of wine fermentation, R Soc Open Sci., 2017, 4, 170900, DOI: 10.1098/rsos.170900.

17 L. Zhou, B. He and J. Huang, Amphibious fluorescent carbon dots: one-step green synthesis and application for light-emitting polymer nanocomposites, Chem. Commun., 2013, 49, 8078, DOI: 10.1039/c3cc43295e. 
18 S. Mitra, S. Chandra, T. Kundu, R. Banerjee, P. Pramanik and A. Goswami, Rapid microwave synthesis of fluorescent hydrophobic carbon dots, RSC Adv., 2012, 2, 12129, DOI: 10.1039/c2ra21048g.

19 Y. Fan, X. Yang, C. Yin, C. Ma and X. Zhou, Blue- and greenemitting hydrophobic carbon dots: preparation, optical transition, and carbon dot-loading, Nanotechnology, 2019, 30, 265704, DOI: 10.1088/1361-6528/ab0b14.

20 X. Zhang, Y. Zhang, Y. Wang, S. Kalytchuk, S. V. Kershaw and Y. Wang, et al., Color-Switchable Electroluminescence of Carbon Dot Light-Emitting Diodes, ACS Nano, 2013, 7, 11234, DOI: 10.1021/nn405017q.

21 S. Mondal, R. Thirupathi and H. S. Atreya, Carbon quantum dots as a macromolecular crowder, $R S C A d v$. , 2015, 5, 4489, DOI: $10.1039 /$ C4RA14019B.

22 F. Cheng, X. An, C. Zheng and S. Cao, Green synthesis of fluorescent hydrophobic carbon quantum dots and their use for 2,4,6-trinitrophenol detection, RSC Adv., 2015, 5, 93360, DOI: 10.1039/C5RA19029K.

23 B. Wang, A. Song, L. Feng, H. Ruan, H. Li and S. Dong, et al., Tunable Amphiphilicity and Multifunctional Applications of Ionic-Liquid-Modified Carbon Quantum Dots, ACS Appl. Mater. Interfaces, 2015, 7, 6919, DOI: 10.1021/acsami.5b00758.

24 W. Shang, M. Ye, T. Cai, L. Zhao, Y. Zhang and D. Liu, et al., Tuning of the hydrophilicity and hydrophobicity of nitrogen doped carbon dots: a facile approach towards high efficient lubricant nanoadditives, J. Mol. Liq., 2018, 266, 65, DOI: 10.1016/j.molliq.2018.06.042.

25 H.-J. Wang, X. He, T.-Y. Luo, J. Zhang, Y.-H. Liu and X.-Q. Yu, Amphiphilic carbon dots as versatile vectors for nucleic acid and drug delivery, Nanoscale, 2017, 9, 5935, DOI: 10.1039/C7NR01029J.

26 A. Talib, S. Pandey, M. Thakur and H.-F. Wu, Synthesis of highly fluorescent hydrophobic carbon dots by hot injection method using Paraplast as precursor, Mater. Sci. Eng., C, 2015, 48, 700, DOI: 10.1016/j.msec.2014.11.058.

27 V. J. Venditto and E. E. Simanek, Cancer Therapies Utilizing the Camptothecins: A Review of the in Vivo Literature, Mol. Pharm., 2010, 7, 307, DOI: 10.1021/mp900243b.

28 D. Jaque, L. Martínez Maestro, B. del Rosal, P. HaroGonzalez, A. Benayas and J. L. Plaza, et al., Nanoparticles for photothermaltherapies, Nanoscale, 2014, 6, 9494, DOI: 10.1039/C4NR00708E.

29 C. G. Alves, R. Lima-Sousa, D. de Melo-Diogo, R. O. Louro and I. J. Correia, IR780 based nanomaterials for cancer imaging and photothermal, photodynamic and combinatorial therapies, Int. J. Pharm., 2018, 542, 164, DOI: 10.1016/ j.ijpharm.2018.03.020.

30 M. Potara, T. Nagy-Simon, M. Focsan, E. Licarete, O. Soritau and A. Vulpoi, et al., Folate-targeted Pluronic-chitosan nanocapsules loaded with IR780 for near-infrared fluorescence imaging and photothermal-photodynamic therapy of ovarian cancer, Colloids Surf., B, 2021, 203, 111755, DOI: 10.1016/j.colsurfb.2021.111755.

31 Q. Pan, J. Tian, H. Zhu, L. Hong, Z. Mao and J. M. Oliveira, et al., Tumor-Targeting Polycaprolactone Nanoparticles with Codelivery of Paclitaxel and IR780 for Combinational Therapy of Drug-Resistant Ovarian Cancer, ACS Biomater. Sci. Eng., 2020, 6, 2175, DOI: 10.1021/acsbiomaterials. 0c00163.

32 N. S. Rejinold, K. Cherukula, J. H. Ha, I. Park and Y. Kim, Olive Oil-Based Ultrafine Theranostic Photo Nanoemulsions: A Versatile Tumor Maneuvering Nanoplatform for Precise Controlled Drug Release in Tumor and Complete Tumor Eradication Mediated by Photo-Chemotherapy, Adv. Ther., 2019, 2, 1800154, DOI: 10.1002/adtp.201800154.

33 S. Li, J. Johnson, A. Peck and Q. Xie, Near infrared fluorescent imaging of brain tumor with IR780 dye incorporated phospholipid nanoparticles, J. Trans. Med., 2017, 15, 18, DOI: 10.1186/s12967-016-1115-2.

34 C. Jiang, H. Cheng, A. Yuan, X. Tang, J. Wu and Y. Hu, Hydrophobic IR780 encapsulated in biodegradable human serum albumin nanoparticles for photothermal and photodynamic therapy, Acta Biomater., 2015, 14, 61, DOI: 10.1016/ j.actbio.2014.11.041.

35 C. Yue, P. Liu, M. Zheng, P. Zhao, Y. Wang and Y. Ma, et al., IR-780 dye loaded tumor targeting theranostic nanoparticles for NIR imaging and photothermal therapy, Biomaterials, 2013, 34, 6853, DOI: 10.1016/j.biomaterials. 2013.05.071.

36 M. Zheng, Y. Li, S. Liu, W. Wang, Z. Xie and X. Jing, One-Pot To Synthesize Multifunctional Carbon Dots for Near Infrared Fluorescence Imaging and Photothermal Cancer Therapy, ACS Appl. Mater. Interfaces, 2016, 8, 23533, DOI: 10.1021/acsami.6b07453.

37 M. Ortega-Muñoz, P. Vargas-Navarro, F. Hernandez-Mateo, A. Salinas-Castillo, L. F. Capitan-Vallvey and S. Plesselova, et al., Acid anhydride coated carbon nanodots: activated platforms for engineering clicked (bio)nanoconstructs, Nanoscale, 2019, 11, 7850, DOI: 10.1039/C8NR09459D.

38 M. Martí, L. Molina, C. Alemán and E. Armelin, Novel Epoxy Coating Based on DMSO as a Green Solvent, Reducing Drastically the Volatile Organic Compound Content and Using Conducting Polymers As a Nontoxic Anticorrosive Pigment, ACS Sustainable Chem. Eng., 2013, 1, 1609, DOI: 10.1021/sc400271k.

39 N. García, R. Rubio-Presa, P. García-García, M. A. FernándezRodríguez, M. R. Pedrosa and F. J. Arnáiz, et al., A selective, efficient and environmentally friendly method for the oxidative cleavage of glycols, Green Chem., 2016, 18, 2335, DOI: 10.1039/C5GC02862K.

40 F. P. Byrne, S. Jin, G. Paggiola, T. H. M. Petchey, J. H. Clark and T. J. Farmer, et al., Tools and techniques for solvent selection: green solvent selection guides, Sustainable Chem Process, 2016, 4, 7, DOI: 10.1186/s40508-016-0051-z.

41 D. Prat, A. Wells, J. Hayler, H. Sneddon, C. R. McElroy and S. Abou-Shehada, et al., CHEM21 selection guide of classical- and less classical-solvents, Green Chem., 2016, 18, 288, DOI: $10.1039 /$ C5GC01008J.

42 S. G. Pedro, A. Salinas-Castillo, M. Ariza-Avidad, A. LaprestaFernández, C. Sánchez-González and C. S. Martínez-Cisneros, et al., Microsystem-assisted synthesis of carbon dots with 
fluorescent and colorimetric properties for $\mathrm{pH}$ detection, Nanoscale, 2014, 6, 6018, DOI: 10.1039/C4NR00573B.

43 C. Ding, Z. Deng, J. Chen and Y. Jin, One-step microwave synthesis of N,S co-doped carbon dots from 1,6-hexanediamine dihydrochloride for cell imaging and ion detection, Colloids Surf., B, 2020, 189, 110838, DOI: 10.1016/ j.colsurfb.2020.110838.

44 L. L. Shevchenko, Infrared spectra of salts and complexes of carboxylic acids and some of their derivatives, Russ. Chem. Rev., 1963, 32, 201, DOI: 10.1070/RC1963v032n04 ABEH001329.

45 R. Blume, D. Rosenthal, J.-P. Tessonnier, H. Li, A. KnopGericke and R. Schlögl, Characterizing Graphitic Carbon with X-ray Photoelectron Spectroscopy: A Step-by-Step Approach, ChemCatChem, 2015, 7, 2871, DOI: 10.1002/ cctc. 201500344.

46 Q.-H. Wu, A. Thißen and W. Jaegermann, XPS and UPS study of Na deposition on thin film V2O5, Appl. Surf. Sci., 2005, 252, 1801-1805, DOI: 10.1016/j.apsusc.2005.03.134.

47 T. G. Avval, C. V. Cushman, S. Bahr, P. Dietrich, M. Meyer and A. Thißen, et al., Dimethyl sulfoxide by near-ambient pressure XPS, Surf. Sci. Spectra, 2019, 26, 14020, DOI: 10.1116/1.5053099.

48 D. Sun, R. Ban, P.-H. Zhang, G.-H. Wu, J.-R. Zhang and J.-J. Zhu, Hair fiber as a precursor for synthesizing of sulfurand nitrogen-co-doped carbon dots with tunable luminescence properties, Carbon, 2013, 64, 424, DOI: 10.1016/ j.carbon.2013.07.095.

49 Y.-W. Zeng, D.-K. Ma, W. Wang, J.-J. Chen, L. Zhou and Y.-Z. Zheng, et al., N,S co-doped carbon dots with orange luminescence synthesized through polymerization and carbonization reaction of amino acids, Appl. Surf. Sci., 2015, 342, 136, DOI: 10.1016/j.apsusc.2015.03.029.

50 F. Heaney and L. Doyle, Bicyclic 5-5 Systems: Five Heteroatoms 2:3 and Six Heteroatoms 3:3. Comprehensive Heterocyclic Chemistry III. Elsevier, 2008, pp. 189-261. (ISBN 978-008-044992-0).

51 W. Boonta, C. Talodthaisong, S. Sattayaporn, C. Chaicham, A. Chaicham and S. Sahasithiwat, et al., The synthesis of nitrogen and sulfur co-doped graphene quantum dots for fluorescence detection of cobalt(II) ions in water, Mater. Chem. Front., 2020, 4, 507, DOI: 10.1039/C9QM00587K.

52 D. Zhang, Y. Hao, L. Zheng, Y. Ma, H. Feng and H. Luo, Nitrogen and sulfur co-doped ordered mesoporous carbon with enhanced electrochemical capacitance performance, J. Mater. Chem. A, 2013, 1, 7584, DOI: 10.1039/c3ta11208j.

53 Q. Xu, P. Pu, J. Zhao, C. Dong, C. Gao and Y. Chen, et al., Preparation of highly photoluminescent sulfur-doped carbon dots for $\mathrm{Fe}(\mathrm{III})$ detection, J. Mater. Chem. A, 2015, 3, 542, DOI: 10.1039/C4TA05483K.

54 S. Kadian, G. Manik, A. Kalkal, M. Singh and R. P. Chauhan, Effect of sulfur doping on fluorescence and quantum yield of graphene quantum dots: an experimental and theoretical investigation, Nanotechnology, 2019, 30, 435704, DOI: 10.1088/ 1361-6528/ab3566.

55 P. Zhu, D. Lyu, P. K. Shen and X. Wang, Sulfur-rich carbon dots as a novel fluorescent imaging probe for distinguishing the pathological changes of mouse-bone cells, J. Lumin., 2019, 207, 620, DOI: 10.1016/j.jlumin.2018.12.010.

56 R. Ludmerczki, S. Mura, C. M. Carbonaro, I. M. Mandity, M. Carraro and N. Senes, et al., Carbon Dots from Citric Acid and its Intermediates Formed by Thermal Decomposition, Chem. - Eur. J., 2019, 25, 11963, DOI: 10.1002/chem.201902497.

57 A. Daina, O. Michielin and V. Zoete, SwissADME: a free web tool to evaluate pharmacokinetics, drug-likeness and medicinal chemistry friendliness of small molecules, Sci. Rep., 2017, 7, 42717, DOI: 10.1038/srep42717.

58 N. Graf, E. Yegen, T. Gross, A. Lippitz, W. Weigel and S. Krakert, et al., XPS and NEXAFS studies of aliphatic and aromatic amine species on functionalized surfaces, Surf. Sci., 2009, 603, 2849, DOI: 10.1016/j.susc.2009.07.029.

59 J. Xu, A. Avellan, H. Li, X. Liu, V. Noël and Z. Lou, et al., Sulfur Loading and Speciation Control the Hydrophobicity, Electron Transfer, Reactivity, and Selectivity of Sulfidized Nanoscale Zerovalent Iron, Adv. Mater., 2020, 32, 1906910, DOI: 10.1002/adma.201906910.

60 K. Soliman, F. Grimm, C. A. Wurm and A. Egner, Predicting the membrane permeability of organic fluorescent probes by the deep neural network based lipophilicity descriptor DeepFl-LogP, Sci. Rep., 2021, 11, 6991, DOI: 10.1038/s41598021-86460-3.

61 J. A. Jaleel and K. Pramod, Artful and multifaceted applications of carbon dot in biomedicine, J. Controlled Release, 2018, 269, 302, DOI: 10.1016/j.jconrel.2017.11.027.

62 K. Wang, Y. Zhang, J. Wang, A. Yuan, M. Sun and J. Wu, et al., Self-assembled IR780-loaded transferrin nanoparticles as an imaging, targeting and PDT/PTT agent for cancer therapy, Sci. Rep., 2016, 6, 27421, DOI: 10.1038/srep27421. 